\title{
Analysis of implementation maternal care program in independent midwife clinic in Padang
}

\author{
Miranie Safaringga ${ }^{1}$, Hardisman $^{2}$, Yulizawati $^{3}$ \\ 1Program of Magister Midwifery, Faculty of Medicine, Andalas University, Indonesia \\ 2Department of Public Health and Community Medicine, Faculty of Medicine, Andalas University, Indonesia \\ 3Departement of Midwifery, Faculty of Medicine, Andalas University, Padang, Indonesia
}

\begin{tabular}{l}
\hline SUBMISSION TRACK \\
\hline Recieved: 20 Juni 2018 \\
Final Revision: 26 Juni 2018 \\
Available Online: 30 Juni 2018 \\
KEYWORDS \\
\hline $\begin{array}{l}\text { Maternal care program, labour, independent } \\
\text { midwifery clinic } \\
\text { CORRESPONDENCE }\end{array}$ \\
\hline
\end{tabular}

Phone: 081372290021

E-mail:miraniesafaringga@gmail.com

\begin{abstract}
A B S T R A C T
Factors that cause maternal mortality mostly focused on the physical factors like post partum bleeding, eclamsia, sepsys, and abortion complication. However, non physical factor like psychological and emotional factor rarely discussed. In fact, such factor also has important role in determine maternal mortality by causing several complication. The government has created several movement to overcome maternal mortality that caused by psycholgical and emotional factor. The movement are known as "Gerakan Asuhan Sayang Ibu". The movement has 18 requirement to fulfill in order to prevent maternal mortality. This study aims to analyze the implementation of "Gerakan Asuhan Sayang Ibu" by Independent Midwife Clinic (BPM - Bidan Praktek Mandiri) in Padang municipality. This study uses qualitative design. Data were collected by deep interview. The study are is conducted in several BPM in Padang Region on december 2017 until february 2018. There are 3 midwives, 3 patients, and 3 family member of patient were particiapate as informant. The data analysis was performed using Triangulation method. The study shown that more than half midwife do the "Asuhan Sayang Ibu" well. The item that related to physical needs are fulfilled by mostly midwife. But the item that related to communication and emotional needs only fulfilled by several midwife. The deep exploration about management of "Asuhan Sayang Ibu" found the planning, implementation and evaluating phase did not run well properly.
\end{abstract}

\section{INTRODUCTION}

Maternal care is a program that can help a safe and secure process, allowing habits, practice and trust to help, and improve performance and health. Many complications and disorders that cause maternal and infant mortality can be 
avoided if labor is well managed. Everyone should be attended by the officer in charge quickly and difficult complications. Maternal care in the process of childbirth is a grouping performed by midwives mingle with mothers and families to provide services with full respect and care according to the needs of mothers and create mutual trust in order to carry out midwifery care. ${ }^{1}$

Normal labor can occur by 5 factors, namely strength or which is the strength or contraction produced by the mother's uterus, the passenger is the fetus itself in the form of fetal size, fetal roots and fetal position, then through the birth canal is the opening of the cervix, perinium stiffness and other factors is the psychic mother. Very helpful in dealing with maternal problems and post-saline mothers. Based on research conducted by Fatmawati found that there is a significant influence between the factors that affect the number of people involved with 2.44 times opportunities for postpartum postpartum with high social numbers. $^{2}$

As Sherer discloses in Henderson that aspects of care that are proven to affect the feelings of labor include communication, information delivery, pain management, the environment of childbirth, the social support of the spouses and of the carers. ${ }^{3}$

More and more evidence suggests that events around labor can make women unable to control their bodies. This results in a very stressful experience that can cause psychological problems such as post traumatic stress disorder (PTSD). It is alleged that birth itself may be a major driver of PTSD or may make women who have previously survived stress experienced trauma. $^{3}$

Essential Competencies for Basic Midwivery Practice issued by International Confederation of Midwives (ICM) 2013 describes the basic competencies that midwives in the world must have, some of which are in line with the implementation of maternal care. ICM stresses that midwives should have the necessary knowledge and skills of midwifery, neonatology, social sciences, public health and ethics that form a high-quality foundation.

In this competence it is mentioned that midwives should respect their culture and customs, regardless of status, ethnic or religious beliefs, maintain privacy, communicate important information between health providers or other family members only with explicit permission from the mother and work in partnership with mothers and their families, enable and support them in making informed choices about their health, including the need for referrals when health care needs exceed the ability of midwives, and their right to refuse testing or intervention. Other competencies described are the principles of interpersonal communication with and support for women and / or families. ${ }^{4}$

Emotional support can include communication skills, information sharing, and counseling skills. Many patterns are now in place to provide care during labor and to facilitate continuity of care providers and to empower families. However, the effectiveness of the program in the service of maternity has not been fully evaluated.3

Midwife attitudes work in expectation to be more positive, and patients do not show any increased stress. Therefore, the care pattern during giving treatment during labor can have a positive effect on mother and midwife. The type of support provided by midwives and family members during labor has long-term effects on a woman's life. Midwives have the authority to ensure that women have adequate support in a supportive environment. ${ }^{5}$ This is very important in efforts to reduce maternal and neonatal mortality since most deliveries in Indonesia are still occurring at the primary health care level, where maternal deaths are not only occurs because of a technical nature, but also some things like psychic that may continue as a predisposing factor of maternal death

Therefore it is still in need monitoring monitoring evaluation of mother care that 
given the midwife to the patient. The evaluation is not only limited to the competence in terms of medical service actions, but also from various aspects including in terms of providing emotional support, safety and comfort in patients.

The research conducted by Yani on the mother of the second stage of maternity said that given maternal care As much as $60 \%$ of respondents perinatal faster $<1$ hour, this proves there is influence of mother care giving to the old labor stage of II. $^{6}$

Based on the above background, researchers are interested in conducting research to analyze the implementation of maternal care in mothers of maternity in the midwife independent practice in Padang Municipality in 2017.

\section{METHOD}

This research is a qualitative study, and data analysis by means of triangulation. This study examines the implementation of maternal care based on the stages of health management by conducting in-depth interviews on each of the informants. Consideration in determining the type of qualitative research, because the problem of this research concerning the actions and behavior between midwives, patients, and patient families. This research was conducted in Midwife Practice Mandiri Padang City, involving 9 informants, namely 3 Midwives, 3 maternity patients, and 3 family of maternity patients. This research was conducted in December 2017 to February 2018.

\section{RESULT}

\section{Planning}

Planning undertaken by the implementing midwife at BPM can not be separated from the things that ensure maternal care can be done well that includes the patient's pregnancy and the extent to which midwives know the patient, midwives preparation, patient relationships and patient families and referral clinics.
Based on the midwife's exposure, it was found that midwife knew her patient, this would support maternal care, if the midwife knew the patient it was easier for the midwife to approach the patient. With regard to preparation, midwives are eligible for adequate delivery such as having sufficient room and delivery equipment.

Based on observations that researchers do on some BPM, there are still no taffel wash at all and there are some have a taffel wash but can not be used.

However, for the nutritional needs of the patient, the midwife should provide before and after delivery, from the observation findings that the midwife only provides feeding after the birth.

The patient's nutritional needs before it is critical to the delivery process because the patient will need a lot of energy to deliver. In addition to the above for the planning care mother affair midwife must know about the closeness of patients with the patient's family. Based on the results of interviews with midwives, it was found that the relationship of patients with the patient's family was good. Maternal care is not only about the relationship or the actions of the midwife to the patient, but also how the midwife with the patient's family because in this case the patient's family acts as a party that can provide a sense of security and comfort during the birth process. In the implementation items are also mentioned about the preparation of referrals for the safety of patients and their babies during the labor process. The referral plan has not been well enough, marked with the midwife not having a fixed referral hospital and never providing or appointing a donor whenever required.

Based on triangulation analysis, it is found that planning in the implementation of maternal care is prepared by the midwife from the place up to the relationship that exists between midwives and patients and their families. From the results of the document review found that the delivery of patients is not in accordance with the estimates. This may lead to a lack of 
midwifery preparation in welcoming patients who will come up with nearcomplete opening, so that the preparation of the tool, the place to the fulfillment of the patient's nutrition before delivery is not met. As a result mother care affection is not achieved properly.

Preparation of the room is good but beberara there is no hand wash and ventalization enough. This can cause the patient to feel uncomfortable because the air circulation in the maternity is not good. From the results of observations of midwives patient relationship with his family both characterized by the presence of family during the BPM. When referring midwives do not prepare prospective donors for patients.

\section{Organizing}

Organizing maternal care is the relationship between individuals who play a role in the care of mother and how the person performs its role so that the birth patient feel safe and comfortable in labor. This is not separated from the needs of different maternity patients far from usual day. the organization of nursing care midwife midwife must understand how the meaning of family presence during childbirth. This is marked by the attitudes of dependence on maternity patients to their families.

From the results of interviews with 3 maternity patients, they agreed that what they want to accompany when delivery is a husband, as people closest and can be in trust. Likewise with the results of interviews with the patient's family, the patient's husband took the initiative to accompany during the delivery wife. This is in line with maternal care, where in the care of mother, the midwife will teach the husband action action that will make the patient feel safe and comfortable. Based on the results of observation researchers found that almost all husbands accompany the wife during labor take place. For the sake of the realization of maternal care, the family of the patient should know the midwife who helps the delivery of their families, with each other know the expected communication will take place more easily.

Based on the researcher's interview with the patient's family. They said that they know the midwife in the clinic, with this matter expected mother care can be done. Based on the observation, the researchers found that in line, the researchers saw several times the midwife talked to the patient's family.

\section{Implementation}

In order to implement the care of mother care goes well, it is expected that midwives can understand the 18 items of maternal affection implementation. The informants in this study were midwives who had attended Normal Birth Training Training (APN), during which training had been explained about maternal care as one of the essence of normal birth called APN's five red threads. However, based on the results of interviews with researchers informants obtained information that midwives have followed the APN training but do not remember the care of mother care.

Based on the results of interviews with informants obtained information that some informants agree that the care of mother's love should be done completely because the care of mother's love prioritizes security, comfort and satisfaction of the patient during delivery at the clinic. Midwives emphasize that mother care is actually done but there are no guides such as checklists, so there are some important items missed. Based on the results of observations of the implementation of maternal care one of the most often forgotten example is when patients enter the second stage of midwife forgot to encourage patients to try a variety of position meneran so that patients feel comfortable.

In the implementation of maternal care requires the good treatment of midwives to maternity patients, based on the results of interviews with maternity 
patients, patients recognize that patients during the clinic interact with many midwives or midwife assistants who practice at the clinic. Maternity patients admit that many are served by the midwife's assistant, so that at the time before delivery the patient feels uncomfortable and lacks confidence so worried that later lead the persalinanya is an inexperienced midwife assistant.

Preparation and behavior of the midwife determines the satisfaction of the maternity patient, one informant points out that when the patient comes with an almost complete opening the midwife is not ready and becomes hasty in preparing the tool and place, so the patient feels uncomfortable and becomes increasingly stressed with the conditions he faces.

\section{Evaluation}

Evaluation in maternal care is done by interviewing midwives of midwives, maternity patients and patient families related to the actions and treatment they receive during labor. In addition, researchers also made observations in order to obtain accurate information about maternity care.

Based on the results of in-depth interviews with midwives in BPM obtained information that informants agree to be evaluated but informants argue that should evaluate the satisfaction of maternity patients performed by health agencies such as health centers. The evaluation should be made and agreed upon by the midwife in the working area of the puskesmas. Informants agree that patient satisfaction evaluation at BPM will be useful for development and evaluation materials for BPM itself.

Based on the results of observations of researchers, there are some of the informants who refused to in mintai information related to mother's care. Some midwives think that the evaluation of maternal care will raise the shortcomings of the ministry and facilities at their BPM, thus affecting the reputation of the BPM. Based on the results of in-depth interviews, informants said that the patient response after childbirth was generally pleased with the birth of her baby. Likewise with the patient's family response was happy with the birth. In addition to midwives, related to the evaluation of maternal care, researchers also conducted interviews with maternity patients. Based on the results of interviews researchers get informed about the patient's expectation of her delivery. The first childbirth patient wants a safe delivery and a healthy baby, in addition the patient wants maximum and safe and comfortable midwifery services. 
The informants said that they were concerned with the safety and health of their babies, but informed that midwives were in a hurry and informants said midwives should be able to provide direct encouragement to patients. Other information can be obtained from the IF $2 \mathrm{C}$ informant that he wants the midwife as before, who is always available beside the patient.

Based on observations made by researchers, found similar thing that midwife is not in BPM since the beginning of patient come. The new midwife arrives when the patient has a complete opening. Although the services provided by midwife assistants are satisfactory, patients will feel more comfortable and secure if handled directly by midwives who have BPM because they are considered to be much more experienced and skilled than midwife assistants.

\section{DISCUSSION}

\section{Planning}

From the results of a review of documents conducted by researchers, it was found that patients who will be maternity at this time did not come in the day that has been interpreted earlier. This can lead to a lack of midwife preparation in dealing with maternity patients. Prepared preparations are found in the preparation of the place. Based on observations most midwives have only one maternity room. Maternity room as well as a recovery room (Reecovery Room) for 2 hours at the time of IV childbirth. During the fourth stage of the patient the patient remains in the same bed during delivery. Meanwhile at the same time new patients are coming to the birth with almost complete opening. This makes the care of mother's love can not be done properly.

Based on the results of interviews with informants found that midwives know their patients, this will support the implementation of maternal care, if the midwife knows the patient it is easier for the midwife to approach the patient. So that will be established a good communication between midwives and patients. Nurulita (2016) in his research mentions the intensity of interpersonal communication has a tendency to affect intimate relationship. If the higher the intensity of one's communication with others, the more familiar the relationship will be between the person. ${ }^{7}$

With regard to preparation, midwives are eligible for adequate delivery such as having sufficient room and delivery equipment. Comfortable room, clean and adequate air circulation can make patient and patient's family feel comfortable. Based on observations that researchers do on some BPM, BPM is still found with a maternity room that does not have good air circulation, and no fan. Maternity patients will experience an increase in metabolism that triggers an increase in body temperature that will make the birth patient will feel more vibrant than others.

In accordance with research conducted Nifa found that adequate air circulation support to carry out therapy. Pain caused by a contraction of the uterus, the phase phase through which the patient is complete until the opening becomes painful.8 Pain management is one of the ways used in the field of health to overcome the pain experienced by maternal mothers, one of them is to do breath relaxation techniques in. The deep breathing relaxation technique is one of the techniques in behavior therapy to reduce tension and anxiety.

In connection with the preparation of the delivery of nutritional needs of patients to be one focus that must be met. Midwives should provide before and after del5ivery, from observations found 19 midwives only provide food after delivery. Fulfillment of postpartum nutrition can support the smooth delivery of labor because the calorie needs of the maternity patient increases as a source of energy during labor.

Sulistyawati revealed in her research that feeding and drinking during labor is appropriate, because it provides more 
energy and prevents dehydration (dehydration can inhibit contraction / irregular and less effective) .9 Therefore, encourage mothers to eat and drink during labor and childbirth, encourage the family to always offer snacks and often drink to the mother during childbirth.

Based on the results of research component planning in the implementation of maternal care is still not fully good. Mature and complete planning is expected to make the care of mother's love is done perfectly so that the maternity patient can feel comfortable and safe labor.

\section{Organizing}

The existence of the family is included in the patient's primary needs. In addition to midwives, families in the care of mothers play a role in psychologically labile patients. Maternal care arranges both relationships. In the care of mother's love, indirectly requires inter-personal relationships must work well. Good midwife relationships with patients, midwives' relationships with the patient's family and the relationship between the patient and his or her own family. Good synergy between personal will lead to communication and action that can be a therapy for the patient's own maternity.

Similar to the research conducted by Nurulita related to the relationship between family members. In his research Nurulita revealed that the test results of the influence between the intensity of communication in the family and the level of physical proximity with intimate relationship showed significant results. So there is influence between the intensity of communication in the family and the level of physical proximity with intimate relationship that can be used as capital in communication therapy in maternal mothers.7

Based on the results of the study found that $100 \%$ of patients want their husbands as a companion during labor. This is characterized by the dependency attitudes indicated by maternity patients towards their husbands. The role of midwives in the care of mother to the family of maternity patients is arranged in four items of the implementation of maternal care. Maternal care requires the midwife to ensure the rights of the patient's family in terms of information on the delivery process of his family. In addition, families also need to be supported during the labor process, because it is not uncommon to find families of patients who have worries when the family gave birth.

The role of other midwives set by maternal care is to teach husbands and family members about ways in which they can pay attention and support mothers during labor. Based on the results of research this item only accomplished only $35.9 \%$. Although midwives recognize in depth interviews that they communicate with the patient's family but the communication content that occurs is not related to the care that will be provided. This indicates that most midwives do not pay much attention to the patient's family functionally to the patient. Patient's family ignorance of the actions she has to perform for the fulfillment of maternity needs will make it difficult for the patient's psychic condition.

To realize the implementation of good maternal care for the sake of comfort, safe and patient satisfaction must establish a good relationship between the actors involved in it. It is set in the organizing component, the results of this study found that the organization of maternal care has been running well but has not shown the quality of good relations.

\section{Implementation}

By following APN training the midwife should be familiar with the item and the implementation of mother's care. But from the results of research that researchers do, it was found that midwives have forgotten the care of mother care items. This is the cause of the low number of the implementation of maternal care in BPM. From the interview with the midwife 
in BPM, they said that they did not remember the item but the midwife said that the care of mother's care had been done even without guidance.

The results of observations that the implementation of the implementation of maternity care that has been done midwife today is still less than perfect and many are forgotten. It would be better if the implementation of maternal care is done by using guidance so that mother care affection is really done and achieved safe, comfortable childbirth and satisfaction for maternity patients and their families.

From the observations found eleven items of care that mother has not been done. The highest item found on item number four is "Encourage mother to ask and talk about fear and worry" that is as much as $87,2 \%$. At the time of observation the researchers found that midwives did not encourage mothers to discuss the concerns that are being experienced by birth patients. This is because the midwife is not in the delivery room for a long time to accompany the patient.

According to the patient's admission when in-depth interviews the patient is only accompanied by an inexperienced midwife assistant, and the midwife only arrives when the opening of the birth canal is complete.In line with the mother care item number eight "Teach husband and family members about ways they can pay attention and support mothers during labor" that is not performed as much as $64.1 \%$ midwife. The lack of attention of midwives to the families of maternity patients is characterized by lack of interaction of midwives with their husbands or families. Based on the results of interviews with the families of the patients, they acknowledge the midwife present only at a certain moment, so that the patient and the patient's family feel less noticed by the midwife at BPM.

Maternal care demands good treatment and touch from midwives to maternity patients, based on interviews with maternity patients, patients acknowledge that patients during the clinic interact with many midwives or midwife practitioners practicing at BPM. This may lead to decreased maternity satisfaction, as the patient has the perception that midwives and midwives 'assistants do not yet have the same ability to serve patients compared to the BPM owners' own midwives. This lack of confidence will trigger the increasing worry of the delivery patient.

The existence of a midwife is not only a caregiver but a midwife is expected as well as a companion during the delivery process.

From the observation of the researchers, and the results of interviews with the bidam assistants, they said that the midwife was not in BPM at that time because it was still within hours of service and there was a need to go elsewhere. So the patient is entrusted to the assistant. So that the patient and the patient's family do not get as the appropriate action with the care of mother's care. as in the mother care item number 5 "Listening and responding to mother's questions and concerns" that did not occur in almost half of the midwives $(46.2 \%)$.

So it can be concluded the main cause of the implementation of some unfulfilled mother care items with extreme findings is the lack of participation and the presence of midwives in assisting during the first stage of childbirth in because by midwives who are still busy with other affairs outside the BPM, so tend to be cuek with patients maternity 1 . The presence of midwives in accompanied by the implementation of maternal care is expected to make the maternity patient becomes more calm and comfortable in facing persalinannnya.

Effect of midwife assistance on the level of pain in active maternal phase mother in Sleman DIY. The results showed that the group of mothers who were given midwife assistance in the active phase had a lower mean pain value of 2.6 scales compared to the mean of pain value in the group who were not provided with midwife assistance. 
In addition to midwives, the existence of the family, especially the husband becomes the determining factor for the achievement of safe and comfortable childbirth. Maternal care makes the husband or family a vital part of this labor process. The existence of the husband is not enough just in the word "present" only, but the husband or family in charge of an active role as a provider of psychotherapy to maternity mothers. The results showed that item number seven is the midwife recommends that the mother to be accompanied by husband and / or family member during childbirth has been done by $74,4 \%$ midwife. With the presence of a companion is expected to reduce the worry of maternity patients.

In addition to providing direct mentoring to maternity patients, motherly care also arranges for referral plans. Referral action will be performed by the midwife if found in maternity condition with the complication. Referrals are often emergency so as to ensure safe and comfortable childbirth for maternity care patients arranging a referral plan in its implementation item, item 17.

Based on the observations, preparing a good referral plan is not implemented in more than half of midwives, namely $82.1 \%$ of midwives. In obstetrics a comprehensive referral plan is required on the fulfillment of some indactors, ie midwives' assistance during referrals, preparation of tools or partus sets for any time required during the referring trip, vehicle to refer, referral certificate as a legal requirement for referral hospital administration, medications that are needed during referral, family assistance and the need for preparation of potential donors that may be required by the patient at any time. However, when observing the observation, the researcher found that the indicator was the least prepared by the midwife is the donor candidate, which was marked with the patient's KIA book, the blood group was not filled by the midwife during the ANC examination, so it was not known to the patient group and the prospective donor

Based on the results of interviews with midwives, some respondents said they did not cooperate with referral hospitals, so it is necessary to find a hospital with a gynecologist who standby in the hospital. So it can trigger a delay which results in patient discomfort. According to the informant, the lack of cooperation is due to the hospital

Based on the results of midwife research in BPM Padang city is still not good in doing mother care. Therefore the midwife should be able to carry out the care of mother's affection in full so that the profession of midwife remains an option for the community in handling delivery.

\section{Evaluasi}

Evaluation in maternal care is done by interviewing midwives of midwives, maternity patients and patient families related to the actions and treatment they receive during labor. Evaluation or assessment activities are an important part of the management process and are based on management information systems. Evaluation carried out because of the encouragement or desire to measure the achievement of work or program implementation activities against the goals that have been set. Evaluations will provide feedback on the program or implementation of an activity.11 Without an evaluation, it is difficult to know the extent to which objectives that have been planned by a program have been achieved or not.

Evaluation is seen as a way to improve decision-making for future actions.

In the process of evaluation the researchers also made observations in order to obtain accurate information about mother's care. To answer the specific evaluation component of the midwife, the investigators explored information about midwives' views on the evaluation of maternal care during delivery at the clinic. Interview with midwife in BPM get during this evaluation of mother care not yet 
implemented but midwife said that they agree to do evaluation of mother care by institution or authorized institution like puskesmas. In the future, midwife performance needs to be evaluated in order to see whether the work outcome is appropriate or not with the standard of care of normal delivery. Midwife performance is very influential on satisfaction of maternity patient service.

Interviews by midwives recognize that evaluation is important for the progress and development of their BPM. Some midwives found there refused to be observed, they refused from the beginning the researchers came to BPM to make a deal, the agreement made was the midwife would be willing to contact the researcher if there is a patient who will give birth to the BPM belonging to the midwife. Refusal to observe indicates that there is still a midwife who closes with development. Although some midwives say that the services they provide are good but not necessarily in accordance with the standards so it is necessary to evaluate.

Maternity care makes the maternity patient as the center of care, comfort, equality and satisfaction of the birth patient becomes the determining factor of the success of the care provided. The results of the study found that 3 informants were not satisfied after delivery, therefore the patient wanted the midwife's performance can be evaluated periodically. In the dissatisfaction of maternity patients have hopes for midwives in BPM in delivery care services. Maternity patients expect midwives to accompany them during childbirth like the old midwife their parents told. While accompanying midwives are expected to perform some relaxation techniques to make patients comfortable and pain less.

This is reinforced by the results of research conducted by Nabb MT, et al mentions that back massage and breathing techniques can reduce the intensity of pain in both maternal primigravida and multigravida mother from the previous score $8.5-7,5$ to an average of 6.6 on scale visual analogues, thereby reducing pharmacological use of therapy.12 For the accompaniment patients performed by will bring a feeling of calm and comfort.

Based on observations made by researchers, found similar thing that midwife is not in BPM since the beginning of patient come. The new midwife arrives when the patient has a complete opening. Although the services provided by midwife assistants are not too bad, patients will feel more comfortable and safe if handled directly by midwives who have BPM because they are considered more experienced and skilled than midwife assistants.

\section{CONCLUSION}

Comprehensive care for maternal care was found that maternal care items related to physical needs such as infection prevention, respect for mother's privacy and others were done well by more than half of the midwives, but with regard to mother's communication and emotions such as; provision of information, advice to ask about maternal and other concerns is only done by less than half the midwives in the city of Padang. In-depth information on maternal care management at the planning, implementation and evaluation stage has not been good, but at the organizing stage it is'good. 


\section{REFERENCES}

Fatmawati AD. Influential Risk Factors Against Postpartum Blues Occurrences. Journal of Edu Health 2015 Sep: 05 (2): 82

Henderson, C. Jones, K. Essential Midwifery, $1^{\text {st }}$ ed. Translator: RiaAnjarwati, RenataKomala Sari and Dian Adiningsih. Jakarta: EGC, 2008

Health Education Center.Antenatal Care. Jakarta: Education Center for Health Personnel, 2003. 15

Hunt C Sheila. Symonds A. The Social Meaning of Midwifery. Jakarta : EGC; 2007

Hartati, D. Hidayat, A. Effect of Midwifery Assistance to Pain Rate on Maternal Maternity Active Phase at LPTP-KIA Sleman District of Yogyakarta Special Region (thesis). College of Health Sciences' Aisyiyah Yogyakarta; 2013

International Confederation of Midwives. Essencial Competencies for Basic Midwivery Practice, Netherlands : 2013.

Nurulita, D. Influence of Intensity of Communication in Family and Physical Proximity Level towards Intimate Relationship. Journal of Communication Science. 2017

Nifa, MK. Ambarwati. Giving Technin Relaxation Breath In to PASien Kala I Labor in Maternity Room General Hospital of Kudus District.Journal of Nursing Professions.2016: 3 No. 2

Rifana K.I. Betrix. Evaluation of Public Health Programs. Journal of Malang State University: 2015

Sulistyawati, A., Nugraheny, E. Midwifery Care In Maternity Mother. Jakarta: SalembaMedika; 2010

Yani, PD. Wulandari, TD. (2014). The Influence of Maternity Waiting for Maternal Wife Against the Prolonged Births of Primipara Kala Ii. Jakarta: Journal of Eduhealth. 2014; 4 (Pt1): 101-4

\section{BIOGRAPHY}

The first author is a midwife. She had completed his midwifery diploma III education at the Alifah Padang Midwifery Academy in 2012. Then she continued her diploma education IV for educator midwifery and completed it in 2013. In 2013 the author obtained a practice permit to become an practice midwife in one of the midwifery clinics in Padangpanjang city. in 2015 the author continued her education in the postgraduate program in midwifery in the Medical Faculty of Andalas University and successfully completed in 2018. The author currently works as a lecturer at the Alifah Padang Midwifery Academy. Second author is a lecture in Department of Public Health and Community Medicine, Faculty of Medicine, Andalas University. He had done his graduate as Doctor of Medicine (MD), Faculty of Medicine, Andalas University, Padang, Graduated in 2003. Then he continued at Master of Health and International Development (MHID) at Flinders University, Adelaide, Australia in 2008. then in 2012 he was done his study as Doctor of Public Health (DrPH), Flinders University, Adelaide, Australia. He is expert at Healthcare ethics, community and family medicine practice, international health and development and Medical and health professional education. The third author had completed his midwifery diploma III education at the Padang health polytechnic in 2004. Then he continued his midwifery diploma IV education at Padjajaran University in Bandung. After that he continued his education at postgraduated program midwifery at Padjajaran University in Bandung and was completed in 2010. She is currently a lecturer in the undergraduate midwifery program in Department of medicine Andalas University. 